\title{
Rediseño de rutas de transporte urbano aplicando sistemas de información geográfica caso: Riobamba
}

\section{Redesign of urban transport routes applying geographic information systems case: Riobamba}

Oscar Omar Espindola Lara. ${ }^{1}$, Fabian Patricio Londo Yachambay ${ }^{2}$, \& Narcisa de Jesus Sanchez Salcan. ${ }^{3}$

\begin{abstract}
.
The Geographic Information Systems (GIS) and its application in various planning topics such as the transport of belongings and products, GIS have essentially focused on the mobility of people in the urban environment. At present, the problem for government entities is: to promote the movement of citizens, optimize routes, improve frequency times and guarantee the quality of public transport. The research is based on the theoretical principles of transport geography, explores concepts of accessibility and spatial mobility to indicate trends in residential activity, such as the center and some peripheral areas of the city of Riobamba. The investigative process was based on the discovery of data, starting with the extraction of information, then the collection and classification of geographic files, data tables and information provided by entities of the sectional government, which were processed and transformed to be represented in maps of: population density, transport routes and educational infrastructure density. Results were obtained that allowed to identify the imbalance in both the spatial distribution, density of transport routes and the insufficient integration of the service. Bus lines mostly converge to the city center, with low levels of response times depriving users of decentralized and inclusive mobility. To associate the nodes and arcs connected to the transport network, the Graph Theory was used and, based on

\footnotetext{
${ }^{1}$ Universidad Nacional de Chimborazo. Facultad de Ciencias de la Educación Humanas y Tecnologías oespindola@unach.edu.ec

${ }^{2}$ Escuela Superior Politécnica de Chimborazo, Facultad de Administración de Empresas. Riobamba, Ecuador.flondo@espoch.edu.ec

${ }^{3}$ Universidad Nacional de Chimborazo. Facultad de Ciencias de la Educación Humanas y Tecnologías nsanchez@unach.edu.ec
} 
the morphological characteristics of the city, network analysis was applied for the design of the new transport routes, interconnecting the new nodes and centralities, integrating the new routes, guaranteeing users adequate mobility to public and private services, located in the city of Riobamba

Keywords: Mobility, urban transport, transport network

\section{Resumen.}

Los Sistemas de Información Geográfica (SIG) y su aplicación en diversas temáticas de la planificación como es el caso del transporte de pertenencias y productos, los SIG se han centrado esencialmente en la movilidad de las personas en el entorno urbano. En la actualidad, la problemática para entidades gubernamentales es: impulsar el movimiento de los ciudadanos, optimizar las rutas, mejorar los tiempos de frecuencia y garantizar la calidad del transporte público. La investigación se fundamenta en los principios teóricos de la geografía del transporte, explora conceptos de accesibilidad y movilidad espacial para señalar las tendencias de actividad en núcleos residenciales, como en el caso del centro y algunas zonas periféricas de la ciudad de Riobamba. El proceso investigativo se fundamentó, en el descubrimiento de datos, iniciando por la extracción de información, luego la recopilación y clasificación de archivos geográficos, tablas de datos e información proporcionados por entes del gobierno seccional, los que fueron procesados y transformados para ser representados en mapas de: densidad poblacional, rutas de transporte y densidad de infraestructura educativa. Se obtuvieron resultados que permitieron identificar el desbalance tanto en la distribución espacial, densidad de rutas de transporte y la insuficiente integración del servicio. Las líneas de autobuses en su mayoría confluyen al centro de la ciudad, con bajos niveles de tiempos respuesta privando a los usuarios de una movilidad descentralizada e integradora. Para asociar los nodos y arcos conectados a la red de transporte se utilizó la Teoría de Grafos y, a partir de las características morfológicas de la ciudad, se aplicó análisis de redes para el diseño de las nuevas rutas de transporte, interconectando los nuevos nodos y centralidades, integrando las nuevas rutas, garantizando a los usuarios una movilidad adecuada a servicios públicos y privados, localizados en la ciudad de Riobamba.

Palabras claves: Movilidad, transporte urbano, red de transporte

\section{Introducción.}

El mundo se urbaniza rápidamente y la densidad poblacional aumenta. Un informe de las Naciones Unidas estima que aproximadamente el $70 \%$ de la población mundial vivirá en ciudades en el 2050 (Houghton, Reiners y Lim, 2009). Este crecimiento conlleva una expansión de la demanda a la que están sometidas todas las infraestructuras urbanas, incluyendo el transporte. (Houghton et al., 2009).

El Libro Blanco del Transporte publicación realizada por la Comisión Europea (2011) de la Dirección General de Movilidad y Transporte describe que la calidad y accesibilidad de los servicios de 
transportes son aspectos que ganarán una creciente importancia en los próximos años, entre otras cosas debido al envejecimiento de la población y a la necesidad de fomentar el transporte público.

El actual sistema de transporte público en Riobamba, Ecuador, fue adaptado acorde al desarrollo periférico. Cuando se reasentó la nueva ciudad, se planteó la distribución homogénea de la población, la geometría vial en forma de malla permitió que el proceso de urbanización se armonice para el futuro crecimiento.

Actualmente la asignación de las líneas o rutas de transporte urbano se acoge a la expansión progresiva y proliferación de nuevos asentamientos, el crecimiento longitudinal (de Noroeste a Sureste) marca el recorrido de los primeros autobuses y la transformación del territorio. Las cooperativas de transporte están asignadas a rutas específicas, para superar la distancia que separa a la población de los diversos centros de actividad (bancos, municipio, iglesias, escuelas).

Con la ampliación de la zona urbana, la demanda de movilización ha crecido de forma exponencial. Sin embargo, el servicio de transporte público es deficiente, en lo que concierne a la cobertura, tiempo de respuesta, frecuencias con grandes intervalos de espera, la escaza señalización de las paradas, la insuficiente información de los trazados. La asignación de nuevas líneas de autobús o el incremento de rutas, se han ejecutado empíricamente, acorde a la solicitud dirigida al departamento de movilidad, por moradores de los barrios; poco se ha considerado la demanda, áreas de cobertura e infraestructura vial.

\section{Metodología}

La metodología de investigación se respalda en el proceso de descubrimiento en base de datos conocido por sus siglas en inglés como KDD (Knowledge Discovery in Databases). Esta práctica de análisis es aplicado en la minería de datos para extraer la información necesaria en investigaciones con grandes volúmenes de datos, siendo una técnica flexible y de interacción entre el humano y el ordenador (Pérez y Santín, 2008).

Swift (2002) afirma que la expresión descubrimiento del conocimiento en bases de datos, se emplean con el sentido de extracción y transformación de datos; otras aseveraciones se refieren a la extracción de datos como una forma de exploración, diseño y presentación de la información.

Esta metodología permite, recopilar la información, identificar las fuentes, integrarla y con el apoyo de herramientas computacionales, procesar los datos; además dependiendo del tipo de proceso, en este caso análisis geográfico, la presentación de la información es versátil, acogiendo las necesidades del investigador.

En la Figura 3-2 se presenta el esquema de la metodología aplicada; la información será procesada mediante herramientas de análisis cartográfico, seleccionando las áreas de estudio, cargando los datos en la tabla de los shapefile, identificando los centroides de las zonas censales, y clasificando la infraestructura de servicios de la ciudad. 
Para evaluar el actual escenario en relación a las rutas de servicio se parte del análisis de los patrones de movilidad y la red que en conjunto conforma todo el sistema de transporte urbano (Díaz et al.); esta evaluación permite comprender la dinámica de expansión de la ciudad, los procesos segregativos, y los requerimientos de desplazamiento (Garretón, 2012).

Otro aspecto para la evaluación del actual sistema de transporte es la generación de indicadores espaciales, estos se enfocan en múltiples visiones, tanto para una visión global para la urbe en estudio, integrando rasgos individuales básicos y la heterogeneidad del comportamiento de los habitantes (Hernándes et al., 2016).

Para determinar el nivel de accesibilidad del transporte público y la distribución espacial se identifica las principales actividades urbanas (Ruilova, 2012), estas actividades emergen de las necesidades que tienen los individuos como colectivo y la interacción entre distintas acciones, estas se interconectan conformando una red que articula y dinamiza la ciudad.

El acceso a los servicios públicos y privados es fundamental para el desarrollo de las urbes, esta accesibilidad se establece por la localización de los equipamientos y la distribución de la población (Gutiérrez y García, 2002),

Las áreas de mayor concentración poblacional se condiciona por la densidad y distribución espacial de las actividades económicas de la población, esto determinará la dispersión de los destinos que incrementan la necesidad de movilidad (Pablo et al., 2003); aplicando la zonificación espacial se identifica donde existe mayor concentración de habitantes distribuidas en el territorio urbano, en conjunto con la separación de actividades, para nuestro caso salud y educación (Pozueta , 2000). Al zonificar el territorio, se identifica la dimensión espacial, con respecto a la distribución y localización de los equipamientos urbanísticos (Soria-Lara et al., 2014)

\section{Resultados}

De acuerdo al modelo metodológico utilizado para el rediseño de rutas de transporte urbano de la ciudad de Riobamba, una vez trazadas e identificadas las 16 líneas actuales, en la Tabla 1 se obtuvo la longitud en kilómetros, el área de cobertura aplicando un buffer de 235 metros, y el tipo de trazado, sea longitudinal o transversal.

Tabla 1. Longitud en kilómetros

\begin{tabular}{lccc}
\hline Nombre & Longitud km & $\begin{array}{c}\text { Cobertura buffer de 235m } \\
\text { área } \mathbf{~ k m}^{\mathbf{2}}\end{array}$ & $\begin{array}{c}\text { Tipo de } \\
\text { trazado }\end{array}$ \\
\hline Línea 1 & 23.51 & 7.39 & Longitudinal \\
Línea 2 & 21.81 & 7.42 & Longitudinal \\
Línea 3 & 23.94 & 6.35 & Longitudinal \\
Línea 4 & 21.43 & 6.77 & Longitudinal \\
Línea 5 & 30.65 & 9.02 & Longitudinal \\
Línea 6 & 18.71 & 6.09 & Longitudinal \\
Línea 7 & 26.06 & 7.54 & Longitudinal \\
Línea 8 & 17.88 & 4.54 & Transversal \\
\hline
\end{tabular}




\begin{tabular}{lccl}
\hline Línea 9 & 26.07 & 8.94 & Longitudinal \\
Línea 10 & 23.46 & 8.33 & Longitudinal \\
Línea 11 & 17.61 & 5.17 & Longitudinal \\
Línea 12 & 21.49 & 5.40 & Transversal \\
Línea 13 & 18.75 & 4.79 & Longitudinal \\
Línea 14 & 32.94 & 10.89 & Longitudinal \\
Línea 15 & 19.60 & 6.42 & Longitudinal \\
Línea 16 & 27.93 & 7.47 & Longitudinal \\
& & $\mathbf{1 1 2 . 5 3}$ & \\
\hline
\end{tabular}

En la matriz evidenciamos las 16 líneas de transporte de la ciudad de Riobamba, su longitud y cobertura que son ejecutadas por cada una de las mismas y el trazado que tienen.

Tabla 2. Líneas de trasporte

\begin{tabular}{llcl}
\hline & Longitud & Cobertura & \\
\cline { 2 - 4 } Max & $32.94 \mathrm{~km}$ & $10.89 \mathrm{~km}^{2}$ & Línea 14 \\
Min & $17.61 \mathrm{~km}$ & $4.54 \mathrm{~km}^{2}$ & Línea 11 \\
Media & $23.24 \mathrm{~km}$ & $7.03 \mathrm{~km}^{2}$ & \\
\hline
\end{tabular}

En la matriz se evidencia la longitud máxima, media y mínima; para la longitud máxima con 32.94 kilómetros que corresponde a la línea 14, la distancia mínima recorrida pertenece a la línea 11 con 17.61 kilómetros y la distancia media de 23.24 kilómetros; la cobertura o superficie están en concordancia con los valores antes mencionados.

Gráfico No1. Niveles de concentración

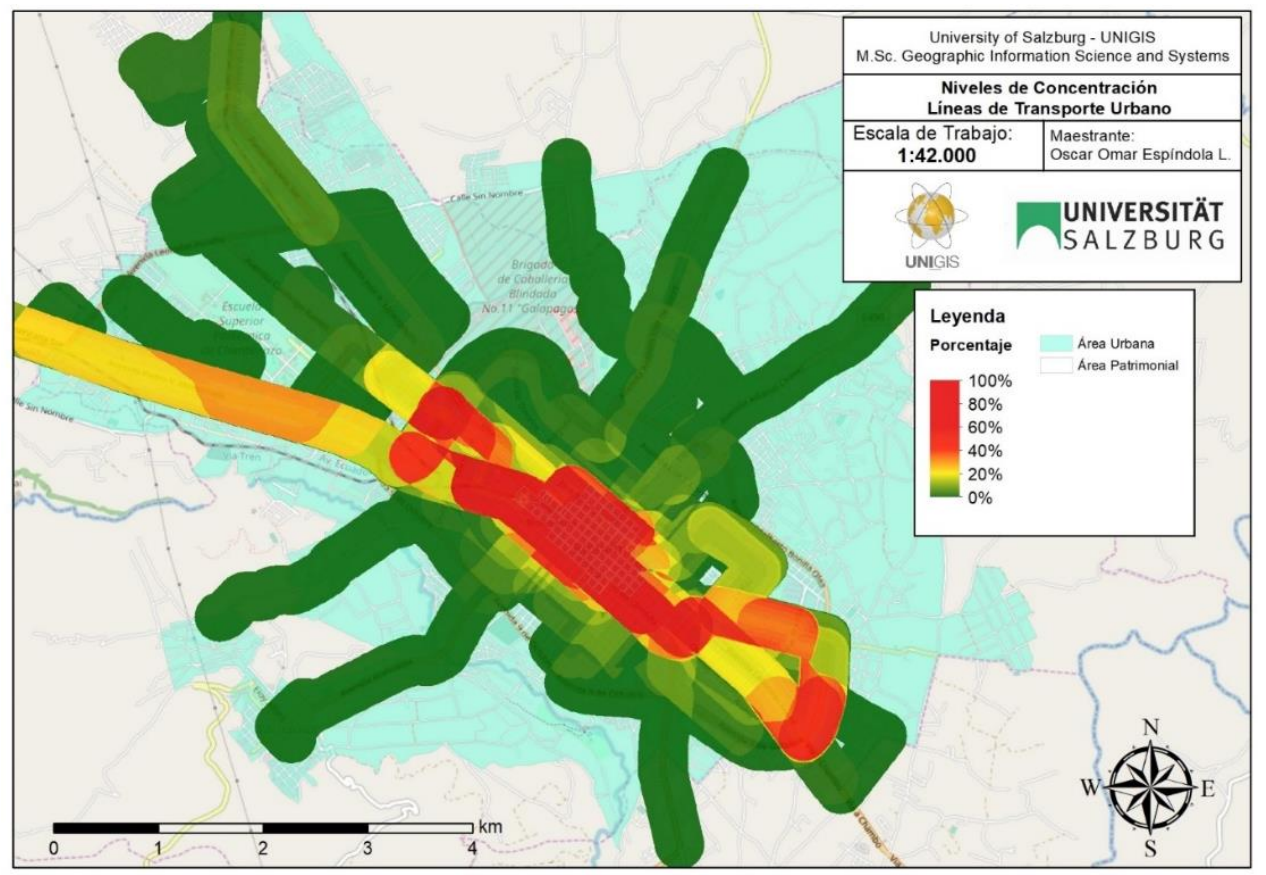


Con el resultado de la unión y conversión a raster de cada uno de los buffers de las rutas de transporte, se observa los niveles de densidad o sobre oferta del servicio, advirtiéndose una alta concentración en el área patrimonial de la ciudad.

Tabla 3. Numero de Líneas

\begin{tabular}{cc}
\hline $\begin{array}{c}\text { Número de líneas que circulan } \\
\text { dentro del buffer de } 235 \mathrm{~m}\end{array}$ & Porcentaje - densidad \\
\hline 16 & $100 \%$ \\
14 & $90 \%$ \\
13 & $80 \%$ \\
11 & $70 \%$ \\
10 & $60 \%$ \\
8 & $50 \%$ \\
6 & $40 \%$ \\
5 & $30 \%$ \\
3 & $\mathbf{2 0} \%$ \\
2 & $10 \%$ \\
1 & $\mathbf{0} \%$ \\
\hline
\end{tabular}

En la siguiente matriz se muestra el número de líneas que circulan dentro del buffer de 235 metros, junto con el porcentaje de densidad, es decir una relación del número de rutas que prestan el servicio dentro de una misma área

Gráfico No2. Líneas que circulan
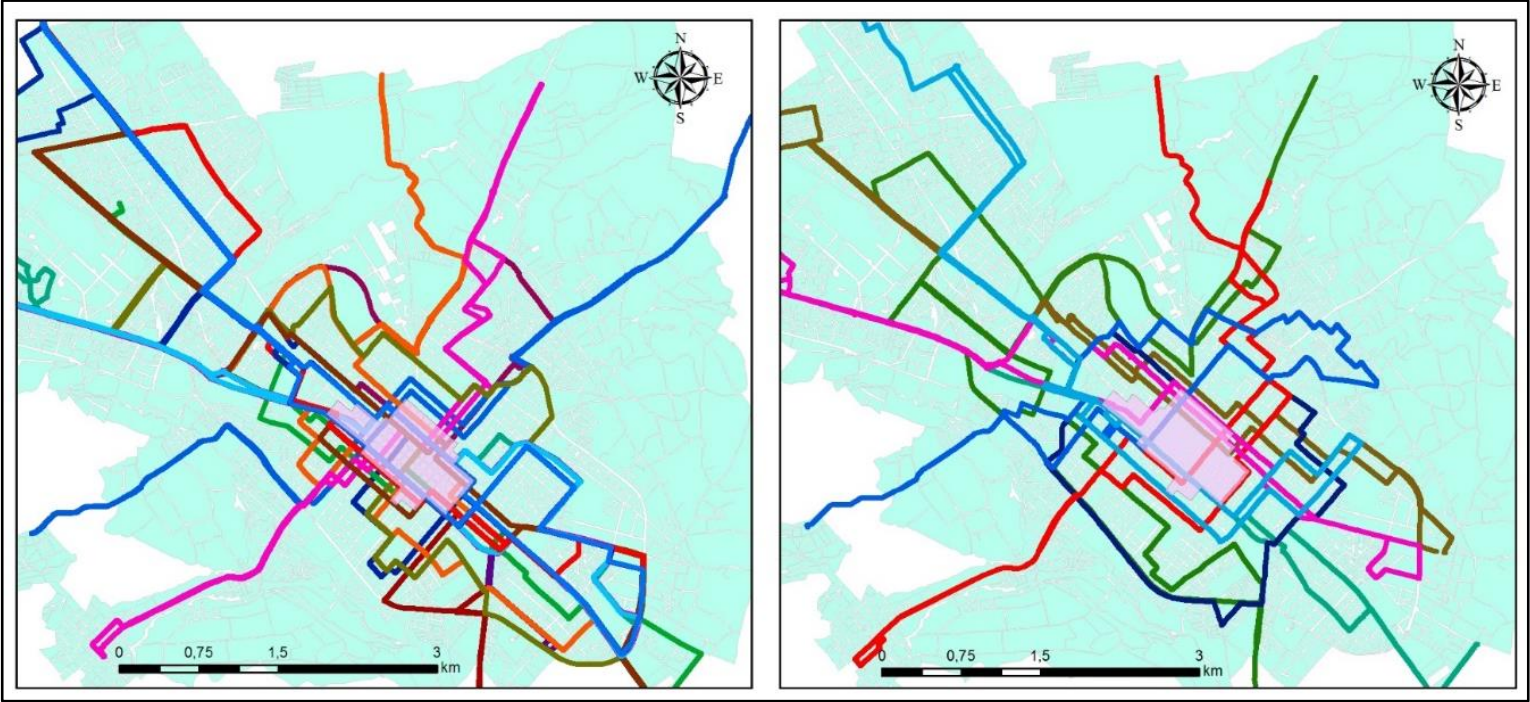
Gráfico No3. Líneas que circulan

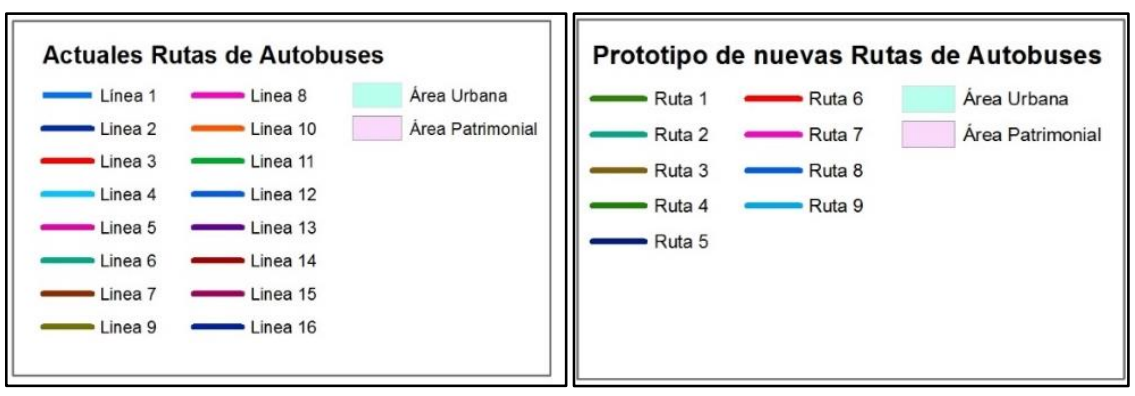

En la Figura se visualiza el trazó de un prototipo o propuesta de rutas de transporte urbano, interconectando a cada una de las centralidades; se tomó en cuenta los establecimientos de alta demanda como: la Escuela Politécnica de Chimborazo y la Universidad Nacional de Chimborazo, paralelamente se disminuyó la carga de líneas de transporte que circulaban en el área patrimonial, por considerarse un sector sensible.

\section{Discusión}

Se debe mejorar los niveles de accesibilidad y calidad del transporte público en la ciudad de Riobamba, la escaza infraestructura respecto a paradas, y áreas de integración de rutas ha incentivado a los pobladores a utilizar el vehículo privado, generando mayor circulación automotriz en la red vial urbana.

Cardozo et al. (2006), en su investigación relacionada al transporte público en la ciudad de Resistencia-Argentina, aplican una metodología similar para determinar las áreas de influencia de las líneas de autobús, estos son presentados como eminentemente descriptivos, sin embargo no carecen de valor, porque evidencian los desequilibrios espaciales en el trazado de las líneas de transporte urbano.

Al determinar la longitud recorrida de cada línea de transporte se podría estimar el costo de las tarifas del servicio, tomando en cuenta otras variables como son: costos de mantenimiento, insumos mecánicos, salario de choferes, entre otros; esto con la finalidad de buscar una mayor productividad y eficiencia de cada operador de transporte.

Para representar la densidad de rutas de transporte, se bosquejó la actual configuración de líneas de autobús, en la cual existe una zona donde todas las líneas se intersecan, si se realiza un análisis comparativo, se concluye que en esa zona hay una densidad de líneas del $100 \%$.

Sin embargo, también se puede interpretar que en esta zona existe integración entre las rutas de transporte, por tanto, es indispensable conocer el área donde confluyen las rutas, en el caso de estudio las 16 rutas circulan dentro del área patrimonial. 
Analizando la cantidad de líneas transversales, se estima la carencia de líneas que atraviesen la ciudad, en tanto, las limitantes de este tipo de rutas, exime a los usuarios movilizarse a zonas ubicadas a los extremos Norte-Este y Sur-Oeste de la ciudad.

Al considerar la Tabla que representa porcentualmente la densidad transporte urbano en relación al número de rutas que prestan el servicio dentro de una misma área; existe un contraste espacial entre la zona de integración de transporte y la zona de alta densidad.

En la zona de integración las rutas coinciden o intersecan en una franja específica generalmente en paradas implantadas en áreas peatonales, y luego toman trayectorias distintas; a diferencia de la zona de alta densidad o sobre oferta, donde las rutas se intersecan en áreas donde las paradas están poco definidas y circulan dentro del mismo buffer o zona de influencia de otras rutas de transporte.

\section{Conclusiones.}

- Dando una perspectiva espacial, podemos mencionar que la aplicación de SIG en el rediseño de las rutas de transporte urbano permitió evaluar la configuración del servicio, la dinámica del actual trazado, su rango de acción, y la cobertura en el área urbana de la ciudad de Riobamba.

- Aplicando buffers a cada ruta de autobús, se evidenció el desbalance espacial en la oferta del servicio de transporte urbano, comprobando que todas las líneas de autobús se concentran en el área patrimonial, zona sensible por las edificaciones que datan del siglo XVIII.

- La concentración de oferta del transporte ha aglomerado la infraestructura de servicios, agrupándolos exclusivamente en el área patrimonial de Riobamba, esto se precisa aplicando herramientas de análisis espacial con respecto a la ubicación de las edificaciones; mientras que en otros sectores consolidados se reducen las posibilidades de instalación de infraestructura que demanda la ciudadanía.

\section{Referencias bibliográficas.}

Alonso, J. M. (2008). Flujo en redes y gestión de proyectos: Teoría y ejercicios resueltos. Oleiros, La Coruña: Netbiblo.

Barrientos, M. (2007). Network Analyst, El análisis de redes desde ArcGis 9.2.

Buzai, G. y Baxendale, C. (2008). Modelos de localización-asignación aplicados a servicios públicos urbanos: Análisis espacial de escuelas EGB en la ciudad de Luján. Revista Universitaria de Geografía, 17(1), 233-254.

Cadena, N. (2015). Plan de desarrollo y ordenamiento territorial 2015 - 2019.

Cardozo, O., Bonfanti, F. y Parras, A. (2006). Los Sistemas de Información Geográfica y la Planificación del Transporte Público: Aplicaciones en la ciudad de Resistencia (ChacoArgentina). Instituto de geografía, 
Castro, J. (2013). Transporte público sostenible en la ciudad de Córdoba, Argentina. Una visión desde la evaluación ambiental estratégica (Tesis de Maestría). Universidad Nacional de Córdoba, Argentina. Recuperado de http://hdl.handle.net/11086/4152

Cendrero, B. y Truyols, S. (2008). El transporte: Aspectos y tipología. Las Rozas, Madrid: Delta.

Comisión Europea. (2011). Libro blanco del transporte: Hoja de ruta hacia un espacio único europeo de transporte : por una política de transportes competitiva y sostenible. Luxemburgo: Oficina de Publicaciones de la Unión Europea.

Conesa, C., Álvarez, Y. y Granell, M. C. (2004). El Empleo de los SIG y la teledetección en planificación territorial. Murcia: Grupo de Métodos Cuantitativos y Sistemas de Información Geográfica, Teledetección; Universidad de Murcia, Departamento de Geografia.

Cuenin, F. y Silva, M. (2010). Identificación y fortalecimieto de centralidades urbanas: El caso de Quito. Washington D.C.

Dávila, P. y Dávila, T. (2008). Tutorial de prácticas de Network Analyst en ArcGis 9.1 y en un Servidor de Mapas (Tesis). Universidad del Azuay, Cuenca. Recuperado de http://dspace.uazuay.edu.ec/bitstream/datos/2349/1/06846.pdf

Díaz Muñoz, M. Á., Cantergiani, C., Salado, M., Rojas, C. y Gutiérrez, S. (2007). Propuesta de un sistema de indicadores de sostenibilidad para la movilidad y el transporte urbanos: Aplicación mediante SIG a la ciudad de Alcalá de Henares. Cuadernos de geografía, (81), 31-49.

GAD Riobamba. (2014). Plan de movilidad de la ciudad de Riobamba.

Ganau, J. y Vilagrasa, J. (2003). Ciudades, arquitectura y espacio urbano. (3), 37-73. Recuperado de http://www.publicacionescajamar.es/publicacionesperiodicas/mediterraneo-economico/mediterraneo-economico-3-ciudades-arquitectura-yespacio-urbano/20/

Garretón, M. (2012). Desigualdad espacial y utilidad social: Esfuerzos de movilidad y accesibilidad en el Gran Santiago. Territorios, 2(25), 36-64.

Hernández, D. y Witter, R. (2011). Entre la ingeniería y la antropología: hacia un sistema de indicadores integrado sobre transporte público y movilidad. Revista Transporte y 
Territorio, Universidad de Buenos Aires, (4). Recuperado el 14/04/2011 de http://www.redalyc.org/html/3330/333027082003/

Houghton, J., Reiners, J. y Lim, C. (2009). Transporte inteligente. Cómo mejorar la movilidad en las ciudades. IBM Institute for Business Value. Estados Unidos: IBM Global Business Services,

Pérez, C. y Santín, D. (2008). Minería de datos: Técnicas y herramientas ( $1^{\mathrm{a}} \mathrm{ed} .2^{\mathrm{a}}$ reimp). Madrid: Paraninfo Cengage Learning.

Pozueta, J. (2000). Movilidad y planeamiento sostenible: Hacia una consideración inteligente del transporte y la movilidad en el planeamiento y en el diseño urbano. Madrid: Instituto Juan de Herrera.

Ruilova, Á. (2012). Red urbana una propuesta alternativa de ciudad. (Trabajo de titulación de arquitecto) (Tesis). Universidad Técnica Particular de Loja, Loja. Recuperado el 23/04/2012 de http://dspace.utpl.edu.ec/handle/123456789/2734

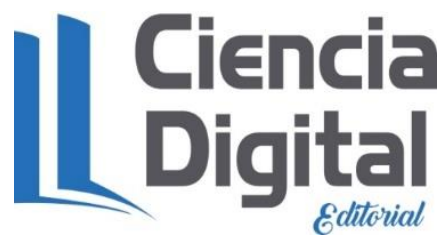




\section{Para citar el artículo indexado.}

Espindola Lara, O., Londo Yachambay, F., \& Sanchez Salcan, N. de J. (2019). Rediseño de rutas de transporte urbano aplicando sistemas de información geográfica caso: Riobamba. Explorador Digital, 3(3.1), 19-29. https://doi.org/10.33262/exploradordigital.v3i3.1.862

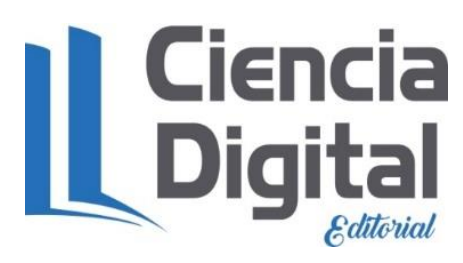

El artículo que se publica es de exclusiva responsabilidad de los autores y no necesariamente reflejan el pensamiento de la Revista Explorador Digital.

El articulo queda en propiedad de la revista y, por tanto, su publicación parcial y/o total en otro medio tiene que ser autorizado por el director o editor de la Revista Explorador Digital.
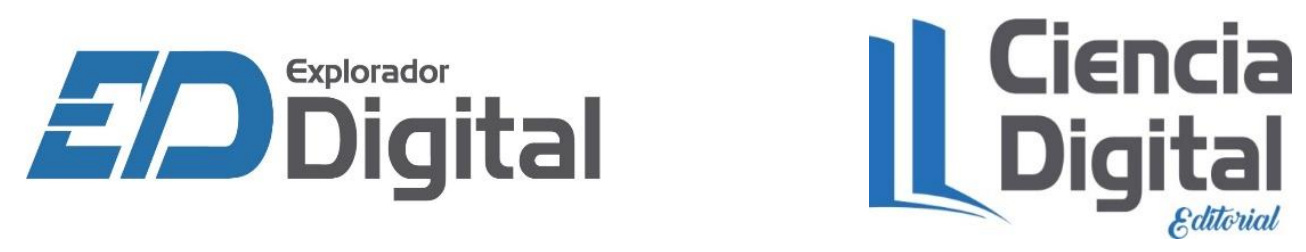